\title{
Design and Implementation of Forest Monitoring System using IOT
}

\author{
Mr.S.Syed Husain ${ }^{1}$, Mr.K.Vigneshwaran ${ }^{2}$, Mrs.S.Kaveya ${ }^{3}$ \\ Assistant Professor, Department of Electronics and Communication Engineering, \\ K. Ramakrishnan College of Engineering, Trichy, India ${ }^{1,2,3}$
}

\begin{abstract}
Traditional approaches to the tracking of wild animals provide useful, yet spatially constrained information. Remote sensing offers the prospect for large area characterizations of biodiversity in a systematic, repeatable, and spatially exhaustive manner. Information and monitoring systems for the forest sector are beneficial for effective policies and planning, valuation of forest resources and proficient investments. This project presents a system for monitoring forest and its vicinity based on IoT based wireless sensor network technology. The need to be able to accurately monitor forest cover and quality is crucial to understanding the costs of deforestation. The monitoring of forest and the surrounding area can, however, still is considered an open research problem due to its substantial vast area. Even though sufficient manpower has been deployed, it is inefficient as it could be life-threatening. This project is an attempt to prevent forest mishaps, the intrusion of animals in the surrounding forest areas, illegal activities in the forest by using wireless sensor technology and eliminating manual power to the highest possible extent.
\end{abstract}

Keywords: Tilt Sensor, Temperature Sensor, Vibration Sensor, Arduino Uno, Wifi Module

\section{INTRODUCTION}

Forests generate water supplies, biodiversity, pharmaceuticals, recycled nutrients for agriculture and flood prevention, and are central to the transition towards a Green Economy in the context of sustainable development and poverty eradication. Recent studies into the extent of illegal logging estimate that illegal logging accounts for 50-90 per cent of the volume of all forestry in key producer tropical countries and 15-30 per cent globally. At home and abroad there are forest fire preventions such as artificial way of looking, forest aircraft, FIRE-WATCH forest fire automatic warning systems and satellite circuit monitoring system, etc. As a result of analysis and comparison with existing systems to monitor logging activities and forest fire explosion based on reconnaissance satellites and remote sensing we have determined several challenges of currently used systems. The over utilisation of ground water has drastically reduced the ground water level in the last 15 years. So it is the need of hour to utilise each and every drop of water wisely so that it can also be used by our coming generations also. Also we should develop some new methods that use the renewable sources of energy. The development of these new techniques are going to reach our goal of sustainable development as well as to cut off the emission of greenhouse gases to a minimum level.

II.

LITERATURE SURVEY

\section{L.K. HEMA,Dr. D. MURUGAN,R.MOHANAPRIYA}

-The prime of the way of exploiting these forest resources is cutting the trees. To conserve the illegal logging of forest wood we proposed a "Wireless Sensor Network based remote forest monitoring and conservation of illegal logging of valuable trees. This system is suitable for large-scale forest monitoring from illegal logging.

\section{GUANGXUE YANG, ZHENG LIU}

- According to the characteristics of forest fire, this paper design a forest fire monitoring system based on wireless sensor network. The system consists of ZigBee coordinator, routers, end nodes and PC computer. The ZigBee nodes compose wireless sensor network by ad-hoc network mode, and is mainly responsible for collecting and transporting the special environment parameters of the forest including temperature, humidity.

\section{SRIDEVI VEERASINGAM, SAURABH KARODI}

- In this paper a portable wireless data logging system for temperature monitoring in real time process dynamics. Process variables (like temperature, pressure, flow, level) vary with time in certain applications and these variations should be recorded so that a control action can take place at a defined set point.

4. Latha. V , S.Omar Farooq, J.Faisal Imraan, K.Jegan Kumar, J.Naveen Kumar.

- The environmental impact of roads is of increasing international interest and concern. The impacts of roads include 
Vol. 8, Issue 5, May 2019

habitat loss, habitat fragmentation and habitat degradation that affect wildlife and its habitats both directly and indirectly especially on larger mammals. These animals have large ranges or undertake seasonal movements over large areas of mainly natural or semi-natural habitat. There has been less attention overall to animals in more modified landscapes with a long history of intensive land use and land management.

\section{PROPOSED WORK}

In the proposed system ,the design uses three sensors tilt sensor(to detect the inclination of tree when its being cut),temperature sensor(to detect forest fires),sound sensor(for effective detection of illegal logging i.e. even the sounds generated while axing the tree are also sensed) Data generated from these sensors is continuously monitored This system can monitor real-time related parameters, e.g., temperature, relative humidity, and send the data immediately to the computer of the monitoring. The collected data will be analyzed and managed by the computer. Compared with the normal meteorological information and basic forest resource data, the system can make a quick assessment of a potential fire danger. With respect to the sensors, their output devices are activated through relay switch. For RF transmitter used in animal body to track and identify to communicate and interface in RF receiver in microcontroller and sound sensor a buzzer is activated and for temperature sensor a water pump is activated.

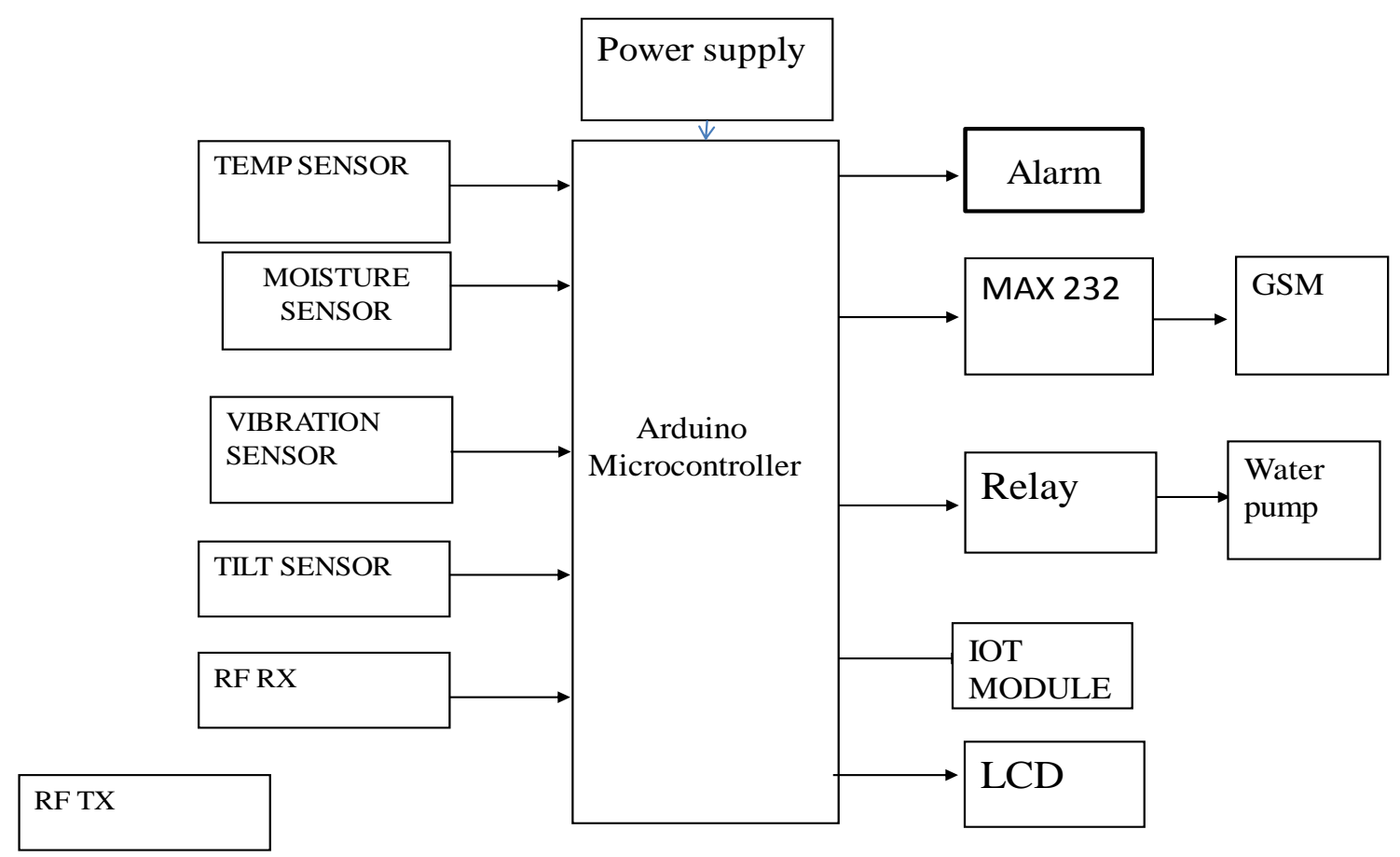

Fig 1. Block diagram of wireless sensor node

\section{HARDWARE IMPLEMENTATION}

A. Soil Moisture Sensors: Soil moisture sensors typically refer to sensors that estimate volumetric water content. Another class of sensors measure another property of moisture in soils called water potential; these sensors are usually referred to as soil water potential sensors and include tension meters and gypsum blocks Measuring soil moisture is important for agricultural applications to help monitoring's manage their forest systems more efficiently.

B. Temperature Sensor: As heat is added to a system, molecular motion increases and the system experiences an increase in temperature. It is difficult, however, to directly measure the energy of molecular movement, so temperature sensors are generally designed to measure a property which changes in response to temperature. The devices are then calibrated to traditional temperature scales using a standard (i.e. the boiling point of water at known pressure).

C. Arduino Microcontroller: The Atmega328 microcontroller in Arduino UNO is used as the brain to control the vehicle tracking system with GPS and GSM module RFID reader. A program is written using C programming language, compiled, and then saved into the flash memory of microcontroller. Arduino UNO board is used to interface the GPS and GSM module. 
D. SIM808 (GPS+GSM+GPRS) module: This module consists of GSM and GPS and GPRS module. It will generates the geographic coordinates and transmits through GSM to the server. The power supply for this module is $12 \mathrm{v}$.

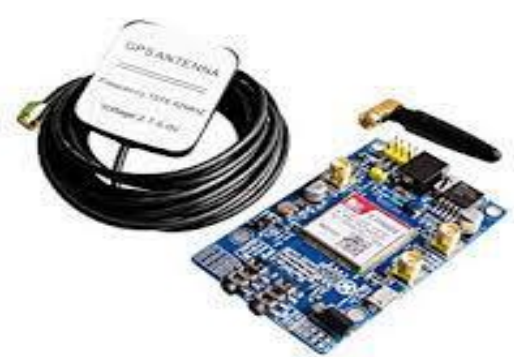

Fig. 2. SIM808(GPS+GPS+GPRS)Module

E. Relay: Relay is an electrical switch using electrical signal to turn on/off the current. It is also used to control a circuit by a low-power signal. On its body, you can see these characters and numbers: 10A 250VAC.Relays are switches that open and close circuits electromechanically or electronically. Relays control one electrical circuit by opening and closing contacts in another circuit. As relay diagrams show, when a relay contact is normally open (NO), there is an open contact when the relay is not energized.

F. DC Motor: A DC motor is any of a class of rotary electrical machines that converts direct current electrical energy into mechanical energy. The most common types rely on the forces produced by magnetic fields.

\section{SOFTWARE IMPLEMENTATION}

A. IOT module:

The Internet of Things (IoT) is the network of physical objects or "things" embedded with electronics, software, sensors, and network connectivity, which enables these objects to collect and exchange data. The Internet of Things allows objects to be sensed and controlled remotely across existing network infrastructure, creating opportunities for more direct integration between the physical world and computer-based systems, and resulting in improved efficiency, accuracy and economic benefit. Each thing is uniquely identifiable through its embedded computing system but is able to interoperate within the existing Internet infrastructure.

\section{B. Arduino IDE Software:}

Arduino IDE software is used for compiling the program into the microcontroller. In this software C programming language has been used for coding. For receiving data from the satellite and RFID reader and sending data into the server, program is written using Arduino IDE software.

\section{Cloud computing:}

Cloud computing, also known as on-demand computing, is a kind of Internet-based computing, where shared resources, data and information are provided to computers and other devices on-demand. It is a model for enabling ubiquitous, ondemand access to a shared pool of configurable computing resources. Cloud computing and storage solutions provide users and enterprises with various capabilities to store and process their data in third-party data centers. It relies on sharing of resources to achieve coherence and economies of scale, similar to a utility (like the electricity grid) over a network. At the foundation of cloud computing is the broader concept of converged infrastructure and shared services.

VI. RESULT AND DISCUSSION

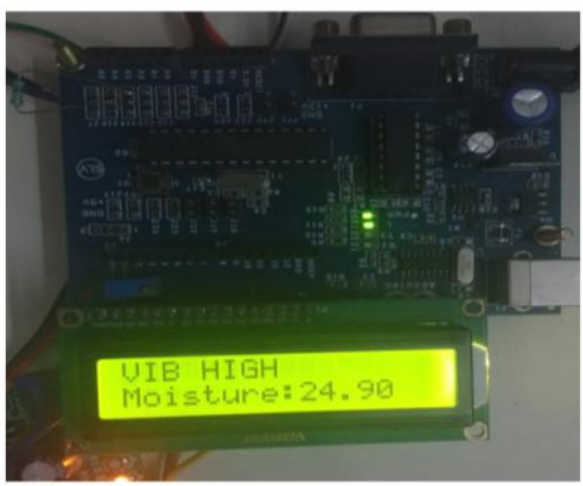

Fig3:Vibration Sensor 


\section{International Journal of Advanced Research in Computer and Communication Engineering}

Vol. 8, Issue 5, May 2019

A sensor that can detect vibration behavior to the machine that is being measured and shown in the fig:3. Various types of vibration sensors are available, but a type called accelerometer is normally used as it offers advantages over other sensors. An accelerometer is a sensor that produces an electrical signal that is proportional to the acceleration of the vibrating component to which the accelerometer is attached.

Fig 4,5 shows the tracking location of Animal in the wildlife reserves or national parks. This project utilizes a GPS modem and a GSM modem for this purpose. Forest officer or Government authority person will get these SMS containing Longitude and Latitude.

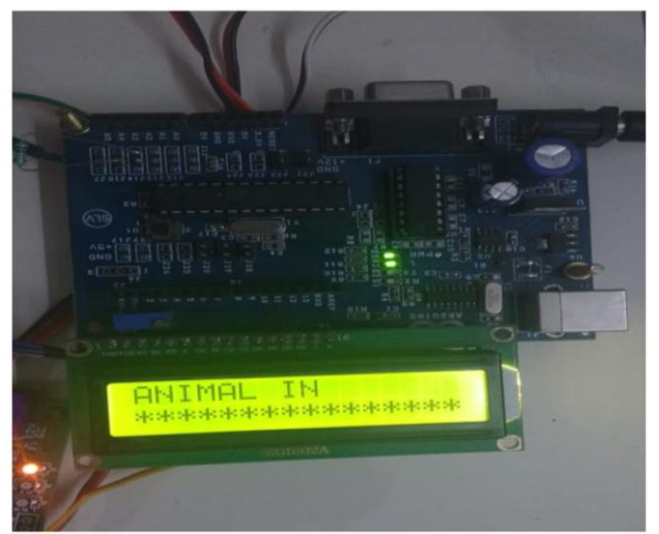

Fig 4:Animal Monitoring

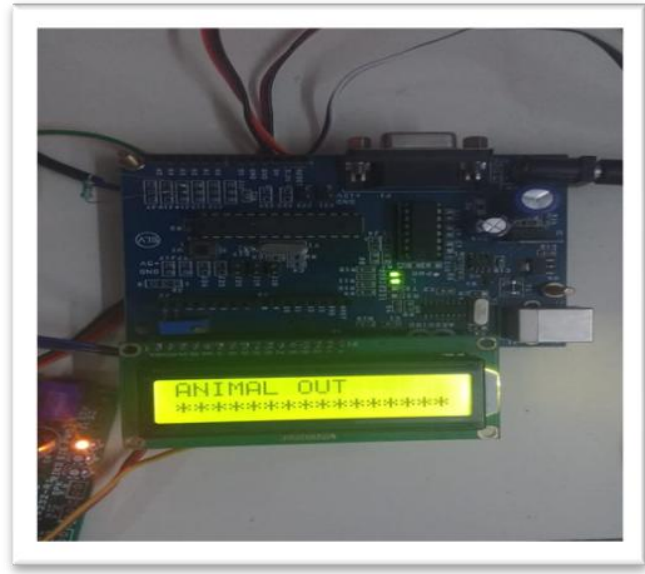

Fig 5:Animal Monitoring

A temperature sensor measures the hotness of an object. Fig 6. shows the temperature rises whenever the voltage increases. The sensor records any voltage drop between the transistor base and emitter.

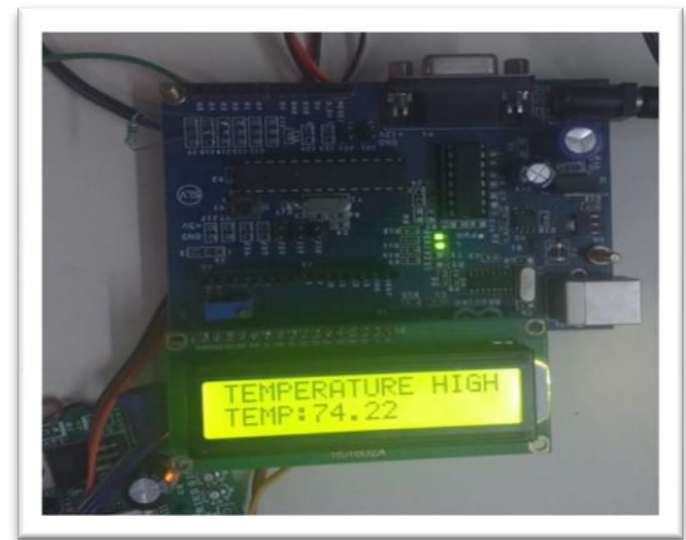

Fig 6:Temperature Sensor 


\section{International Journal of Advanced Research in Computer and Communication Engineering}

Vol. 8, Issue 5, May 2019

It monitors the digital output from the module and makes the decision that it is raining if the digital output goes low. It measures the analog output \& provides a moisture level, where 1023 is high \& where 0 is very wet it is shown in fig 7.

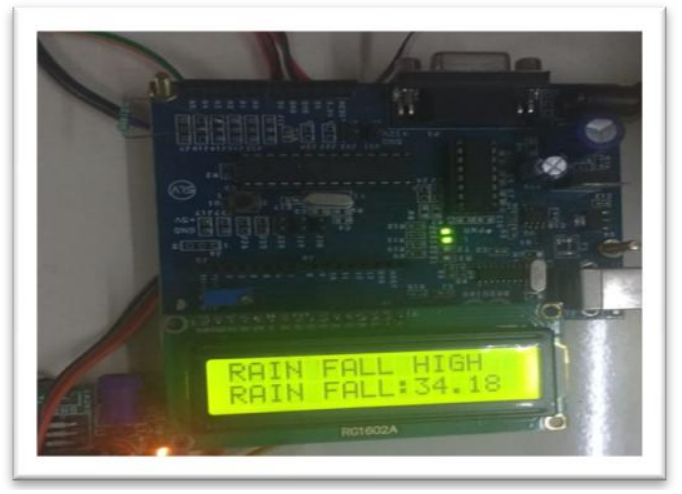

Fig 7:Rainfall Sensor

\section{CONCLUSION}

This paper presents design, development and prototype of wireless sensor network for forest and wildlife monitoring. This prototype system comprises of two modules namely the sensor and the sink module. Here we show the deployment of multisensors needed to cover the forest area. In addition to alerting of forest officials during forest fire accidents and animal monitoring, this system may also be implemented in wildlife sanctuaries and zoos. It can also be implemented as pet tracking system. It mainly targets animal health monitoring, alerting the forest officials during forest fire hazards, smuggling of valuable trees and poaching of endangered species. This system is also highly beneficial in preventing trespassing of wild animals into the living areas in the forest's vicinity. It also aims on animal location and tracking applications. This paper presents a low power consuming, less complex and an economic solution to the existing problem.

\section{REFERENCES}

[1]. A.Flammini, D.Marioli, E.Sisinni, A.Taroni "A real-time wireless sensor network for temperature monitoring" - IEEE Transactions On Industrial Electronics 1-4244-0755-9/07 2007

[2]. F.L.Lewis "Wireless Sensor Networks"- Chapter 4, Smart environments: Technologies, Protocols, and Applications Journal.

[3]. Alan Mainwaring, Joseph Polastre, Robert Szewczyk, David Culler, John Anderson "Wireless sensor networks for Habitat monitoring" WSNA'02, September 28, 2002.

[4]. Mike Horton \& John Suh "A Vision for Wireless Sensor Networks" - IEEE transactions on Industrial Electronics 0-7803-8846-1/05, 2005.

[5]. QiYong. "The development of automatic fire detection technology \& future direction". Fire-fighting Technique \& Product Information, 2002.

[6]. Vivek Kumar Sehgal, Nitin, Durg Singh Chauhan, Rohit Sharma "Smart Wireless Temperature Data Logger Using IEEE 802.15.4/ZigBee Protocol"- IEEE journal, January 2009.

[7]. Liu Yuying, Shi Wangwang. "The design of wireless sensor network based on monitor temperature and humidity by CC2430" Micro computer Information. 2009.

[8]. JI ZhengZhou, Li Yan, Lu Hu "The Implementation of Wireless Sensor Network node Based on ZigBee” - IEEE Journals 978-1-4244-21084/08, 2008.

[9]. P.Ferran, A.Flammini, D.Marioli, "IEEE802.11 Sensor Networking”, IEEE Trans on Instrum \& Meas, vol. 55, no.2, pp.615-619, Apr 2006.

[10]. K.Vighneshwaran, T.Muruganantham, "High Performance Hardware for data detection in Single Carrier Frequency division multiple access (SC-FDMA) based massive MIMO”, International Journal of VLSI and Signal Processing, 2014

[11]. M. Maheswari, T. Margret Rosy, “ Design of an Improved Finite Impulse Response (FIR) filter using Vedic multiplier”, CiiT International Journal of Programmable Device circuits and systems", Vol.7, No.4,pp. 113-118, April-2015

[12]. Awang, A. Suhaimi, M.H.; "A forest monitoring system using wireless sensor networks" IEEE Explorer, ICIAS 2007. 